CARTAS AL DIRECTOR

Localizador web

Artículo 82.578

\section{CONSENSO LATINOAMERICANO SOBRE EL SÍNDROME DEL INTESTINO IRRITABLE: ALGORITMO DE DIAGNÓSTICO Y TRATAMIENTO}

Sr. Director: Recientemente, hemos publicado en su prestigiosa revista «Un consenso latinoamericano sobre el síndrome del intestino irritable», con el objeto de presentar una actualización consensuada sobre la prevalencia, la fisiopatología, el diagnóstico y el tratamiento del síndrome de intestino irritable (SII) dirigido a los pacientes latinoamericanos ${ }^{1}$. El propósito actual es proveer un algoritmo práctico de diagnóstico y tratamiento basado en dicho consenso, de manera que sirva como guía de consulta rápida para los profesionales de la salud que tienen a su cargo el manejo de este problema en nuestra región. La metodología consistió en una discusión inicial que se llevó a cabo en mayo de 2004, en la cual se designó un miembro responsable de la elaboración del documento final. Posteriormente, los miembros del consenso presentaron por escrito sus propuestas sobre el algoritmo, que fueron enviadas por correo electrónico al responsable del documento, quien elaboró un algoritmo inicial consensuado basado en las propuestas. Seguidamente, el algoritmo consensuado se devolvió por correo electrónico para obtener una retroalimentación de los miembros, y surgieron 2 propuestas finales basadas principalmente en el orden de presentación de las recomendaciones del tratamiento. Finalmente, las 2 propuestas se sometieron a votación y por mayoría se obtuvo el algoritmo que presentamos a continuación. Cabe señalar que se utilizan los criterios de Roma II con un escrutinio diagnóstico mínimo inicial, y se indica un cribado para la enfermedad celíaca sólo en las áreas con alta prevalencia de esta enfermedad. El escrutinio inicial permite caracterizar a los pacientes en 2 grandes grupos: $a$ ) los que presentan una edad al inicio del SII mayor de 50 años, signos de alarma o cambio en el patrón de síntomas, los cuales requieren de más estudios diagnósticos, y $b$ ) con una edad de inicio menor de 50 años, ausencia de signos de alarma y patrón de síntomas sin cambios significativos. Estos últimos pueden ser asignados a tratamiento según el síntoma predominante: dolor, distensión/inflamación, estreñimiento y diarrea. Las recomendaciones de tratamiento se presentan en orden descendente según las evidencias disponibles en la bibliografía, desde las más sólidas hasta las menos consistentes, y en todos los grupos se incluye la información y la educación, así como una buena relación médico-paciente, factores básicos en el manejo del SII. Finalmente, en los pacientes refractarios se recomienda una terapia psicológica.

M. SCHMULSON ${ }^{\mathrm{a}}$, J. VALENZUELA ${ }^{\mathrm{b}}$, J. ALVARADO ${ }^{c}$, H. COHEN $^{\mathrm{d}}$, A. DAMIAO ${ }^{\mathrm{e}}$ C. FRANCISCONI ${ }^{\mathrm{f}}$ L. FRUGONE ${ }^{\mathrm{g}}$, J.C. GONZALES ${ }^{\mathrm{h}}$, A. HERNÁNDEZi, B. IADEd, M.H. ITAQUI-LOPES ${ }^{\text {, }}$, R. LATORRE ${ }^{\mathrm{b}}$, J. PRADO P. MORAES-FILHO ${ }^{\mathrm{e}}$, L. SOIFER ${ }^{k}$, M.A. VALDOVINOS ${ }^{a}$, E. VESCO $^{1}$ y A. ZALAR ${ }^{k}$ aDepartamento de Gastroenterología. Instituto Nacional de Ciencias Médicas y Nutrición Salvador Zubirán. México DF. México. 'Facultad de Medicina. Hospital Clínico. Universidad de Chile. Santiago de Chile. Chile. 'Facultad de Medicina. Pontificia Universidad Javeriana. Bogotá. Colombia. 'Hospital de Clínicas Dr. Manuel Quintela. Montevideo.

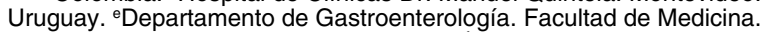
Universidad de São Paulo. São Paulo Brasil. tDepartamento de Medicina Interna. Facultad de Medicina. Universidad Federal de Río Grande do Sul.

Porto Alegre. Brasil. 9Facultad de Medicina. Universidad de Guayaquil. Guayaquil. Ecuador. ${ }^{n}$ Escuela de Medicina. Universidad Central de Venezuela. Caracas. Venezuela. 'Clínicas Multimédica. Ciudad de Guatemala. Guatemala. ${ }^{k}$ Servicio de Gastroenterología del CEMIC. Hospital Juan A. Fernández. Buenos Aires. Argentina. 'Universidad Particular San Martín de Porres. Lima. Perú.

\section{BIBLIOGRAFÍA}

1. Valenzuela J, Alvarado J, Cohen H, Domingo A, Francisconi C, Frugone L, González JC, Hernández A, et al. Un consenso latinoamericano sobre el síndrome del intestino irritable. Gastroenterol Hepatol 2004;27(5):325-43.

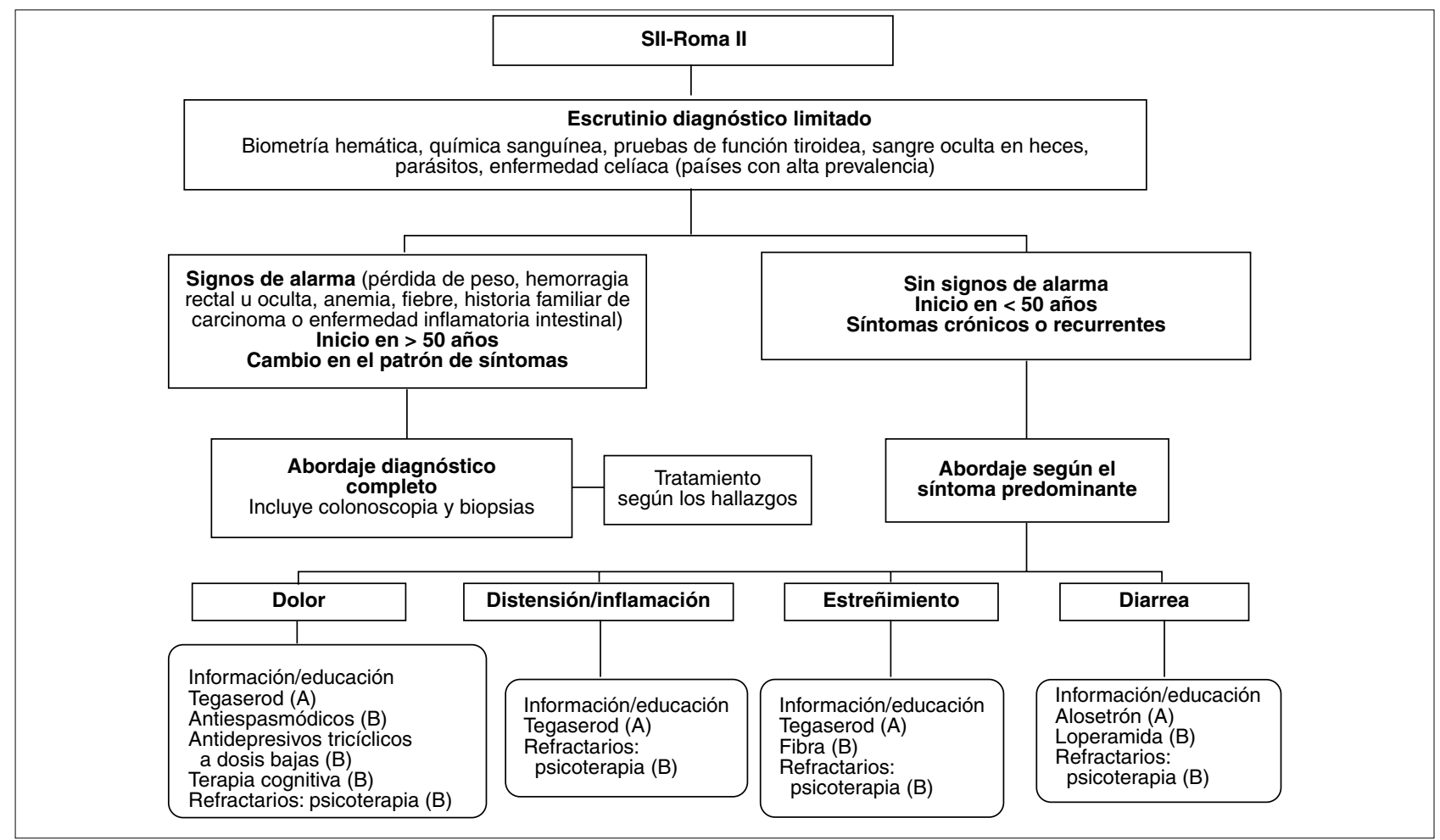

Fig. 1. Algoritmo de diagnóstico y tratamiento para el síndrome del intestino irritable.

Grados de recomendación: grado A, apoyados en evidencias de nivel I; grado B, nivel II; grado C, niveles III-IV. Nivel I, basado en ensayos aleatorizados y controlados con valores de $p<0,05$, con muestras de tamaño suficiente y métodos apropiados; nivel II, basado en ensayos aleatorizados y controlados, con valores de $p>0,05$, ya sea por tamaño insuficiente de la muestra o métodos inapropiados; nivel III, basado en ensayos no aleatorizados con controles contemporáneos; nivel IV, basado en estudios de casos y controles. Antiespasmódicos: bromuro de pinaverio, bromuro de octilonio, trimebutina y mebeverina. 